\title{
Article \\ Unauthorized Unmanned Aerial Vehicle Detection using YOLOv5 and Transfer Learning
}

\author{
Nader Al-Qubaydhi ${ }^{1}$, Abdulrahman Alenezi ${ }^{1}$, Turki Alanazi ${ }^{1}$, Abdulrahman Senyor ${ }^{1}$, Naif Alanezi ${ }^{1}$, \\ Bandar Alotaibi ${ }^{1,2 *}$, Munif Alotaibi ${ }^{3 *}$, Abdelaziz A. Abdelhamid ${ }^{3,4}$, Abdul Razaque ${ }^{5 *}$ and Aziz Alotaibi ${ }^{6}$ \\ 1 Department of Information Technology, University of Tabuk, Tabuk 71491, Saudi Arabia \\ 2 Sensor Networks and Cellular Systems Research Center, University of Tabuk, Tabuk 71491 , Saudi Arabia; \\ b-alotaibi@ut.edu.sa \\ 3 Department of Computer Science, Shaqra University, Shaqra 11961, Saudi Arabia; munif@su.edu.sa \\ 4 Computer Science Department, Faculty of Computer and Information Sciences, Ain Shams University, Cairo, \\ 11566, Egypt; abdelaziz@su.edu.sa \\ 5 Department of Cybersecurity, IITU, Almaty 050000, Kazakhstan; a.razaque@iitu.edu.kz \\ 6 Department of Computer Science, College of Computers and Information Technology, Taif University, Taif \\ 21944. Saudi Arabia, Saudi Arabia; azotaibi@tu.edu.sa \\ * Correspondence: b-alotaibi@ut.edu.sa, munif@su.edu.sa, a.razaque@iitu.edu.kz
}

\begin{abstract}
Recently, the use of drones/unmanned aerial vehicles (UAVs) has notably increased due to their broad commercial spread and low cost. The wide diffusion of drones increases the hazards of their misuse in illegitimate actions such as drug smuggling and terrorism. Thus, the surveillance and automated detection of drones are crucial for safeguarding restricted regions or special zones from illegal drone interventions. One of the most challenging issues in drone detection in surveillance videos is the apparent similarity of drones and birds against complex backgrounds. In this work, an automated image-based drone-detection system utilizing an advanced deep-learning-based objectdetection method known as you only look once (YOLOv5) is introduced for protecting restricted regions or special zones from unlawful drone interventions. Due to the lack of sufficient data, transfer learning was utilized to pretrain the object-detection method to increase the performance. The experiments showed outstanding results, and an average precision of $94.7 \%$ was accomplished.
\end{abstract}

Keywords: drone detection; YOLOv5; unmanned aerial vehicle; deep learning

\section{Introduction}

Drones (also known as unmanned aerial vehicles (UAVs)) can be defined as airplanes without human pilots on board. Drones might work with different levels of independence either under remote control by a human administrator or autonomously with local available PCs [1,2]. Drones were initially utilized for missions excessively risky for people, beginning, for the most part, with military applications [3,4]. In contrast to monitored airplanes, drones are undoubtedly more flexible and can be controlled from anywhere. They provide more prominent perceivability to the pilots operating them since they are directed by satellites and have profoundly advanced cameras.

Recently, drones have rapidly spread and become utilized in various application domains such as sport, agriculture, peacekeeping, surveillance, and transportation [5-7]. Despite the fact that drones are employed to supplant humans in risky missions, they are correspondingly utilized for nefarious purposes. Most importantly, drones can scout targets and collect data from private zones or track moving objects such as humans and vehicles for spying purposes even from remote locations. Additionally, drones can contain weapons for attacking targets or conveying explosives to unpredictably terrorize public places. One of the most important challenges encountered in drone-detection techniques is the presence of birds, which leads to many false alerts. Drone identification is of paramount importance for ensuring the security of restricted areas or private zones. 


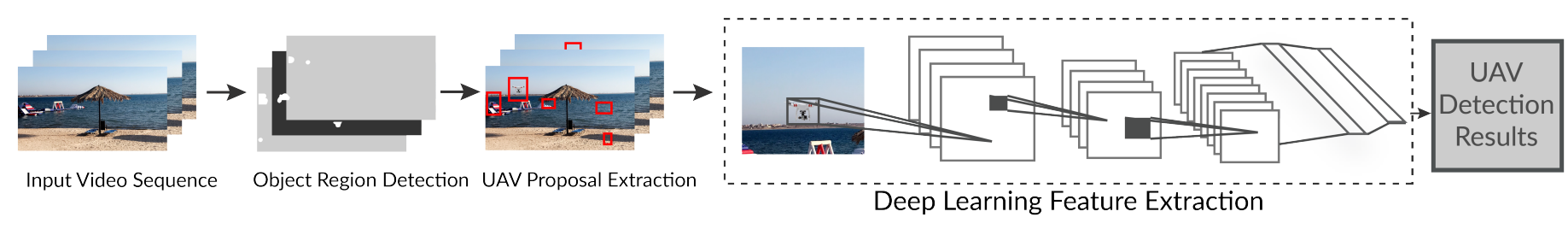

Figure 1. A typical drone detection based on deep learning.

To address these challenges, a drone-detection approach that utilizes an advanced deep-learning-based object detection and recognition method (i.e., a convolutional neural network (CNN)-based technique) is proposed. Object-detection techniques have become more accurate due to the renaissance of deep-learning techniques that reemerged due to powerful computing resources. The aim of this method is to automatically detect objects from videos recorded with diverse contrasts (including low contrast). The proposed method comprises several phases: locating the regions of interest in the image using the selective-search mechanism, extracting the features using $\mathrm{CNN}$, pretraining the model using transfer learning, recognizing the objects using a supervised-learning algorithm, and marking the object's bounding box by integrating the results across the regions. We also included some bird images to ensure that the issue of false alerts due to the coexistence of birds was avoided. To make it more realistic, we inserted some small objects that were hard to locate with the naked eye in the dataset. Figure 1 shows a typical deep-learning-based drone-detection framework using images.

\subsection{Research contribution}

The contributions of this research can be summarized as follows:

- The selective-search mechanism was utilized for image segmentation based on the pixels' intensity to efficiently locate the regions of interest in the images.

- The meaningful features were automatically extracted by adopting the fewer training parameters and adaptability features of the $\mathrm{CNN}$ for obtaining better accuracy.

- Due to the lack of a sufficient number of samples in our dataset, transfer learning to pretrain the model was employed for improving performance.

- We applied a CNN layer to recognize the detected object in the images and marked the object's bounding box through joining the results across the regions.

\subsection{Paper organization}

The remainder of the research paper is organized as follows. Section 2 presents the existing work. Section 4 introduces the UAV-detection model. Section 4 discusses the experimental setup and results. Section 6 concludes our paper.

\section{Related Work}

The previously proposed approaches closely related to our approach are those that detect and differentiate drones from birds present in images captured from videos recorded by static cameras. Saqib et al. [8] proposed a flying-object detection using Faster R-CNN [9] and VGG-16 [10]. The proposed method achieved an acceptable result of $66 \%$ for the mean average precision (mAP). The authors suggested annotating birds as a standalone class to enable the trained model to differentiate birds from drones and improve the performance with regard to reducing false positives.

Aker et al. [11] utilized the you only look once (YOLOv2) [12] framework to differentiate drones from birds after they detected the object in the recorded video. The authors utilized precision-recall (RR) curves to evaluate their method. The recall and precision percentiles both reached 90. Nalamati et al. [13] investigated various approaches to detecting small objects utilizing Faster R-CNN and single shot detector (SSD) [14] and to 
differentiating drones from birds using Inception v2 [15] and ResNet-101 [16]. The authors explored the validity of these architectures using a dataset that contains 8771 frames captured from 11 videos. Two set of experiments were conducted: when the drone was in close proximity to the camera and when the drone was far away from the camera. In the first set of experiments, the results for all the architectures were high. In the second set of experiments, the combination of Faster R-CNN and ResNet-101 performed better in terms of recall and precision than the other architectures. However, the authors omitted the detection time as an effective performance metric when they conducted their experiments and planned to study this metric in future work.

De la Iglesia et al. [19] utilized both a well-known object-detection method (i.e., RetinaNet [20]) and the feature pyramid network (FPN) [21] to predict if the object in the image was a drone or a bird. The FPN was adopted to detect objects on two different levels: the aim of lower pyramidal levels was to detect small objects, while the objective of the upper pyramidal levels was to concentrate on large objects. For recognition, the authors utilized ResNet-50-D [22] and validated this architecture using a dataset that contains images of drones and birds. Magoulianitis et al. [23] used deep CNN with skip connection and network in network (DCSCN) [24] to improve the performance by pre-processing the images. Subsequently, the authors utilized Faster-RCNN to detect the objects. The proposed method achieved acceptable recall results.

Other studies $[25,26]$ divided the drone detection into two phases: the easy-to-detect objects (i.e., objects that were far away from the camera or objects in low-contrast images) belonged to the first phase, while in the second phase, a classifier with high precision was introduced to decrease the number of false positives. Schumann et al. [26] utilized both median-background subtraction and deep-neural-network-based region proposal network (RPN) to develop a robust flying-object (i.e., drones, birds, and clutter) detector. The authors evaluated their method using a customized dataset of 10,286 images. The authors also proposed using VGG-conv5 as a classifier and took first place in the 2017 Drone-vs-Bird challenge. Craye and Ardjoune [25] used two different architectures: the semantic segmentation network U-net [27] for detection and ResNet-v2 for recognition. The proposed method won first place in the 2019 Drone-vs-Bird competition, achieving $71 \%$ recall and $76 \%$ precision.

Seidaliyeva et al. [28] utilized a background-subtraction technique to detect moving objects and a well-known CNN architecture (i.e., mobileNet-v2) to classify the detected objects into three classes: bird, drone, and background. The proposed approach achieved promising results of $70.1 \%$ precision, $78.8 \%$ recall, and $74.2 \%$ f-score. However, the proposed approach's high performance relies on the presence of a moving background.

Khan et al. [29] introduced an end-to-end air-defense system capable of detecting and targeting drones autonomously. The proposed system consists of three phases. The moving objects are detected by radar (i.e., it has the ability to emit microwaves) in the first phase. Once the object is detected, the camera is triggered if any flying object is detected, to recognize what type of object has been detected, using YOLOv3 in the second phase. In the final phase, the laser gun is utilized to lock the threat if the certainty of the recognition exceeds $75 \%$. The proposed detection and recognition model achieved promising average loss results of 0.184961 .

\section{UAV-Detection Model}

\subsection{Background of YOLO Algorithms}

Most of the current high-performance object-detection frameworks are based on $\mathrm{R}-\mathrm{CNN}$ algorithm series and YOLO algorithm series. R-CNN-based object-detection frameworks have achieved high performance in terms of accuracy in various fields; however, these frameworks suffer from slow detection times. In some situations, these frameworks cannot detect objects in real time. To solve the speed problem, a YOLO algorithm series treats the image-detection challenge as a regression problem with a simple cascade model $[30,31]$. The YOLO model is an advanced real-time object-detection model that has gained 
significant attention in the research community and has achieved state-of-the-art performance in various areas. YOLO stands for "you only look once". It is capable of processing a streaming video in less than $25 \mathrm{~s}$.

YOLO divides the 2D images into a grid during the training process. Each cell of the grid is responsible for detecting the object within its border. In target-detection problems, YOLO consider the entire image in the training phase to gain global information. Thus, the entire image is the input to the YOLO network, and the bounding box spot and the category to which the bounding box belongs are returned. The whole image's characteristics are utilized, and the prediction is applied to each bounding box separately. Each bounding box includes five confidences and predictions that are proportional to the grid unit.

The YOLO algorithm was proposed by [32] in 2015. Then, the YOLO algorithm was upgraded to four new versions, namely, YOLOv2 [33], YOLOv3 [34], YOLOv4 [35], and, most recently, YOLOv5 [36,38]. A new training algorithm known as k-means is utilized by YOLOv2 to cluster the bounding boxes. An a priori box is employed to improve the intersection over union (IOU) performance between the ground truth and the prediction box. In the cluster analysis, the distance indicator is represented by the IOU value of the current box and the cluster center box. In comparison with the first version of YOLO, this version achieves better recall and accuracy rates. In YOLOv3, the recognition step is improved due to the utilization of both residual network (ResNet) [16] and Darknet-53. Additionally, feature pyramid networks (FPNs) [21] are utilized to perceive multiscale prediction. These additions to YOLO improved the performance in terms of both accuracy and speed and decreased the false background-detection rate. In YOLOv4, the head part was adopted from YOLOv3, the backbone network was replaced by CSPDarknet-53, and the receptive field was expanded by employing spatial pyramid pooling (SPP) [17] along with path-aggregation network (PANet) [18] in the neck par. These modifications to the architecture improved the feature-extraction ability of YOLOv4 in comparison with the previous algorithms.

\subsection{Our proposed framework}

In this work, we used the most advanced version of the YOLO algorithm series, which is YOLOv5. The YOLOv5 model has a high performance and fast detection speed, and can meet the requirements of real-time applications. It can achieve all the required steps to detect an object using a single neural network very rapidly. In UAV detection, both the detection speed and accuracy are imperative. Thus, YOLOv5 achieves not only outstanding detection performance in UAV detection but also achieves real-time speed. Moreover, it has excellent performance and can easily be trained to detect different objects.

In the YOLOv5 architecture, there are three main components as follows:

- The backbone, which consists of a novel core block named cross stage partial network (CSPNet) [36,38]. CSPNet resolves several issues related to the gradient. It decreases the number of parameters and the number of floating-point operations per second (FLOPS) of the algorithm. Thus, it helps to improve the inference speed and accuracy and shrinks the architecture's size Moreover, in backbone, there are several convolutional layers, four CSP Bottlenecks with three convolutions, and one spatial pyramid pooling fast (SPPF). The main objective of the backbone is to extract differentsize feature maps from the input image by using several rounds of convolution and pooling.

- $\quad$ The neck or the path aggregation network (PAN), which is used for feature fusion. It preserves and passes the features in deep layers to the detection head. Thus, it extract feature information and generates three scales of output feature maps.

- The head or the output part is used for object detection. In the head part, there are several convolutional layers, four CSP bottlenecks with three convolutions, and upsampling and concat layers. The head part predicts image features, creates bounding boxes around the target object, and predicts the class.

The position of the bounding box of the target can be calculated as follows: 


$$
\sigma_{r}^{g}=O_{r, g} * \mho_{T}^{P}
$$

where $r$ is the bounding box of the $g$ grid, and $\sigma_{r}^{g}$ is the confidence score of the $r$ bounding box. $r, g$ is the target if it is within the $g$ box. If the target is in the $g$ box, the value of $O_{i, j}$ would be 0 ; otherwise, it would be $1 . O_{i, j}$ is the intersection over union (IoU), which is a very well-known evaluation metric in image detection. The IOU score depends on the accuracy of the correct location of the bounding box around the target [40].

YOLOv5 itself has several versions, namely, YOLOv5s, yolov5m, yolov5l, and yolov5x. In this work, we used yolov5x. YOLOv5x is the largest model among them. It has 476 layers. These layers create around 87 million parameters.

The feature map of the convolution layer is calculated as:

$$
R=L * W+b
$$

where $L$ is the input volume that convolves $*$ with the trained weight, $W$, of the layer, and $b$ is the bias term. Then, the activation function is applied on output feature map $R$. The output feature map $*$ summarizes the detected features in the input.

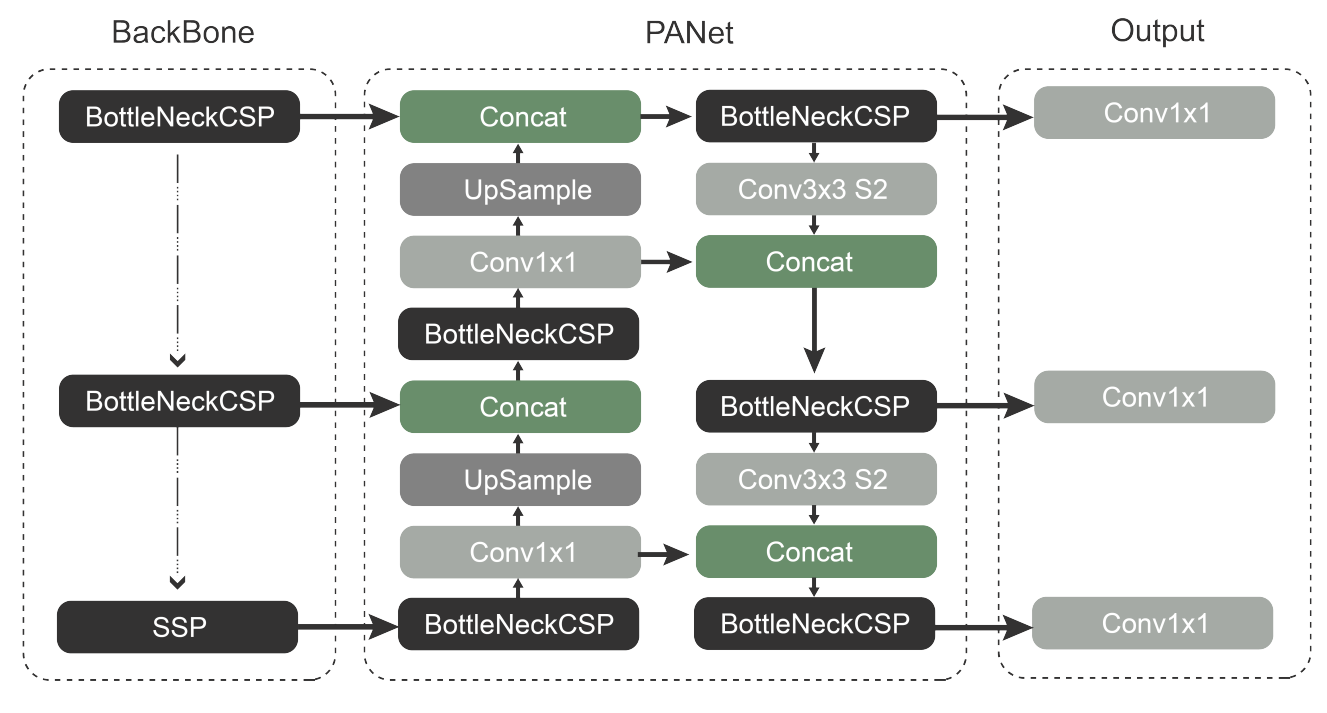

Figure 2. YOLOv5 architecture.

Figure 2 shows the architecture of YoloV5. It shows the backbone, PANet, and the output parts.

There are four layers of feature maps generated in the backbone network. During the training process, YOLOv5x uses data-augmentation steps to cover a broad spectrum of semantic variations. It scales the images by a 0.5 fraction and uses an image translation of a 0.1 fraction.

The loss function, LS, of Yolov5 is calculated as follows:

$$
L S=l_{b x}+l_{S}+l_{j}
$$

where $l_{b x}$ is the regression function for the bounding box, $l_{j}$ is the loss function for the confidence, and $l_{s}$ is the loss function for the classification [43].

The $l_{b x}$ is calculated as follows: 


$$
\begin{aligned}
l_{b x} & =\lambda_{c d} \sum_{i=0}^{s^{2}} \sum_{m=0}^{b} I_{i, m}^{j} b j\left(2-W_{i} \times h_{i}\right)\left[\left(x_{i}-x_{i}^{m}\right)^{2}\right. \\
& \left.+\left(y_{i}-y_{i}^{m}\right)^{2}+\left(w_{i}-w_{i}^{m}\right)^{2}+\left(h_{i}-h_{i}^{m}\right)^{2}\right]
\end{aligned}
$$

where $y_{i}$ and $x_{i}$ are the correct coordinates of the target.

The $l_{s}$ is calculated as follows:

$$
l_{s}=\lambda_{s} \sum_{i=0}^{s^{2}} \sum_{m=0}^{b} I_{i, m}^{j} \sum_{C \in c l} V_{i}(c) \log \left(V V_{i}(c)\right)
$$

The $l_{j}$ is calculated as follows:

$$
l_{j}=\lambda_{n o j} \sum_{i=0}^{s^{2}} \sum_{m=0}^{b} I_{i, m}^{n o j}\left(c_{i}-c_{l}\right)^{2}+\lambda_{j} \sum_{i=0}^{s^{2}} \sum_{m=0}^{b} I_{i, m}^{j}\left(c_{i}-c c_{l}\right)^{2}
$$

where $\lambda_{n o j}$ is the position loss coefficient, $\lambda_{s}$ is the classification loss function, and $c l$ is the classes.

\section{Experimental Setup and Results}

In this section, we present and discuss the experiments conducted to prove the effectiveness of the proposed approach. The section starts with describing the dataset employed in this research, followed by discussing the conducted experiment along with a comparison between the results achieved using the proposed approach and the results achieved using other competing approaches.

\subsection{Dataset}

To detect and recognize drone objects in an image, the machine-learning model should be trained on a set of labeled annotated images. In this research, we employed the freely available drone dataset from Kaggle [39]. This dataset consists of 1359 images of drones captured in a drone-to-earth view. All the images in this dataset were labeled and annotated to fit the training of the adopted Yolov5 framework. The images in this dataset were split into three sets, namely, the training, validation, and test sets. Figure 3 depicts the number of images in the training set along with the distribution of the drone objects in this set. 

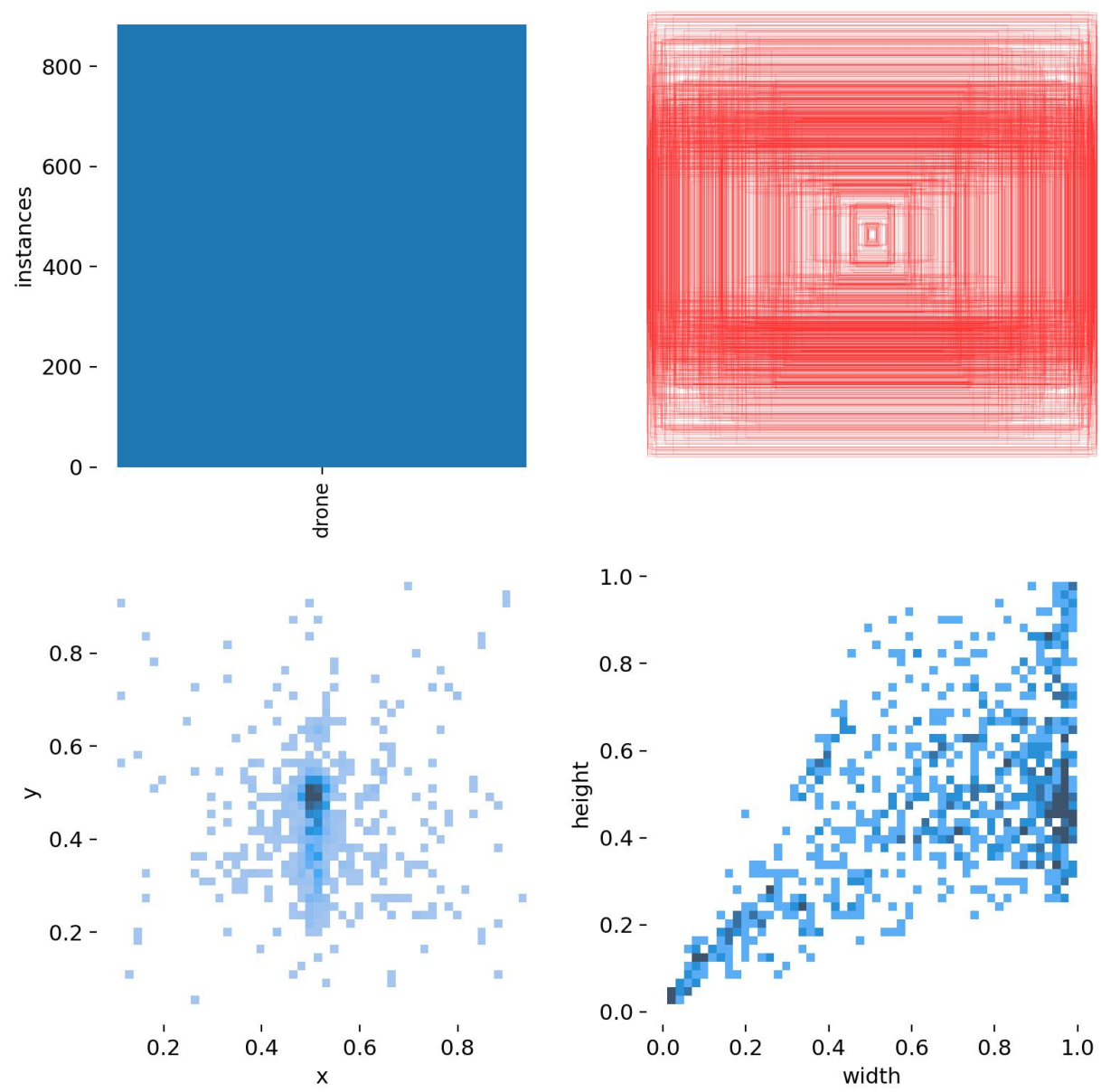

Figure 3. Distribution and histogram of the drone objects in training set.

As shown in Figure 3, the dataset presents a challenge, which is the high variation in the sizes of the drone objects appearing in the images. This variation represents a significant challenge for machine-learning models for accurately detecting and classifying small drone objects against an ambient background. Therefore, a set of masks was generated to look for drone objects in the images. These masks are represented in the figure by the red nested rectangles in the top-right part of the figure.

Figure 4 presents a set of sample drone images in the dataset. As shown in the figure, the dataset contains multiple categories of drones, and the images were captured at different distances from the drone, which represents an advantage of this dataset. In this research, we considered all the categories of drones as a single class.
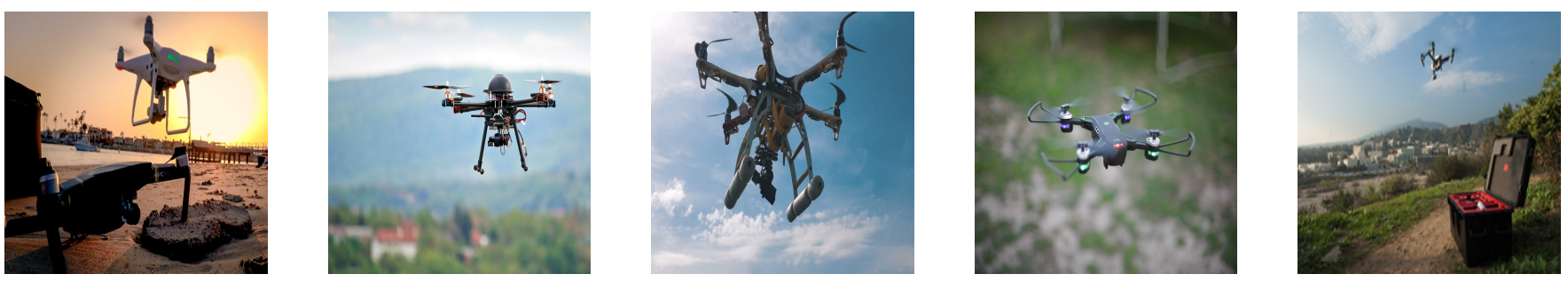

Figure 4. Sample images from the drone dataset.

\subsection{Evaluation Metric}

To evaluate the performance of YOLOv5 trained model in detecting drone, we adopted two metrics namely recall and precision. The curves of these metrics are built by changing 
the threshold of the detection threshold. Therefore, the accuracy of the model can be accurately measured based on these important model evaluation metrics. On the other hands, a distinct and deeper meaning of each of these metrics is presented in the following.

${ }^{*}$ The Precision: This metric measures the percentage of relevant detection results. It can be determined using the following equation:

$$
\text { Precision }=\frac{T P}{(T P+F P)}
$$

where TP and FP denote the number of truly detected objects and the number of nondetected objects, respectively.

*Recall: This metric measures the percentage of total correctly classified results. It can be determined using the following equation:

$$
\text { Recall }=\frac{T P}{(T P+F N)}
$$

where FN refers to the number of falsely detected objects.

For the experiments conducted in this research, if the detected bounding box overlapped by more than $50 \%$ with the predefined bounding box, this detected bounding box was considered a true-positive bounding box; otherwise, it was considered a false-positive bounding box.

\subsection{Transfer learning}

The experiment was conducted using a local machine with an NVidia RTX2070 graphic processing unit (GPU) to train YOLOv5. To run the Yolov5 process on this GPU, cuDNN 7.6.5 was employed. We fine-tuned and configured the YOLOv5 architecture for the Kaggle drone dataset. In this research, we used transfer learning to make the YOLOv5 framework compatible with this dataset. We fine-tuned the last three YOLOv5 and convolutional layers to match the number of classes in the dataset. The original YOLOv5x pretrained model was trained on 80 classes; thus, we changed the number of classes to two, namely, "drone" and "background". To address the data scarcity properly, YOLOv5 introduces various data-augmentation techniques. We fine-tuned the data-augmentation parameters, such as rotation, translation, scaling, and other parameters, to enable YOLOv5 to generate various images from a single image to enrich the given dataset and allow for better training. In addition, the number of batches was set to 4 to increase the robustness of the trained model, and to better match the GPU resources. Finally the number of epochs was set to 100 , after which it was noticed that the trained model became steady and stable. On the other hand, other hyperparameters were incorporated in the conducted experiments, such as decay $=0.00036$, initial learning rate $=0.0032$, final learning rate $=0.12$, and momentum $=0.843$. These parameters were kept with their default values. Finally, we trained and tested YOLOv5 on our local machine using the drone dataset. We trained YOLOv5 for 100 iterations, saved the trained weights for each 25 iterations, and later constructed a number-of-iterations-versus-mAP curve at four different points as weights that had been saved at 25,50,75, and 100 iterations. A flowchart of the overall experiment is shown in Figure 5 .

\subsection{Results}

To evaluate the performance of the proposed fine-tuned yolov5 in detecting drone objects, we utilized the pretrained model weights for transfer learning. These pretrained weights were trained on the COCO large dataset. The training process was then established based on the fine-tuned parameters described in the previous section. The training set was composed of $60 \%$ of the overall dataset images, while the validation set consisted of $20 \%$ of the images in the dataset, and finally, the testing set was composed of the other $20 \%$ of the dataset images. 


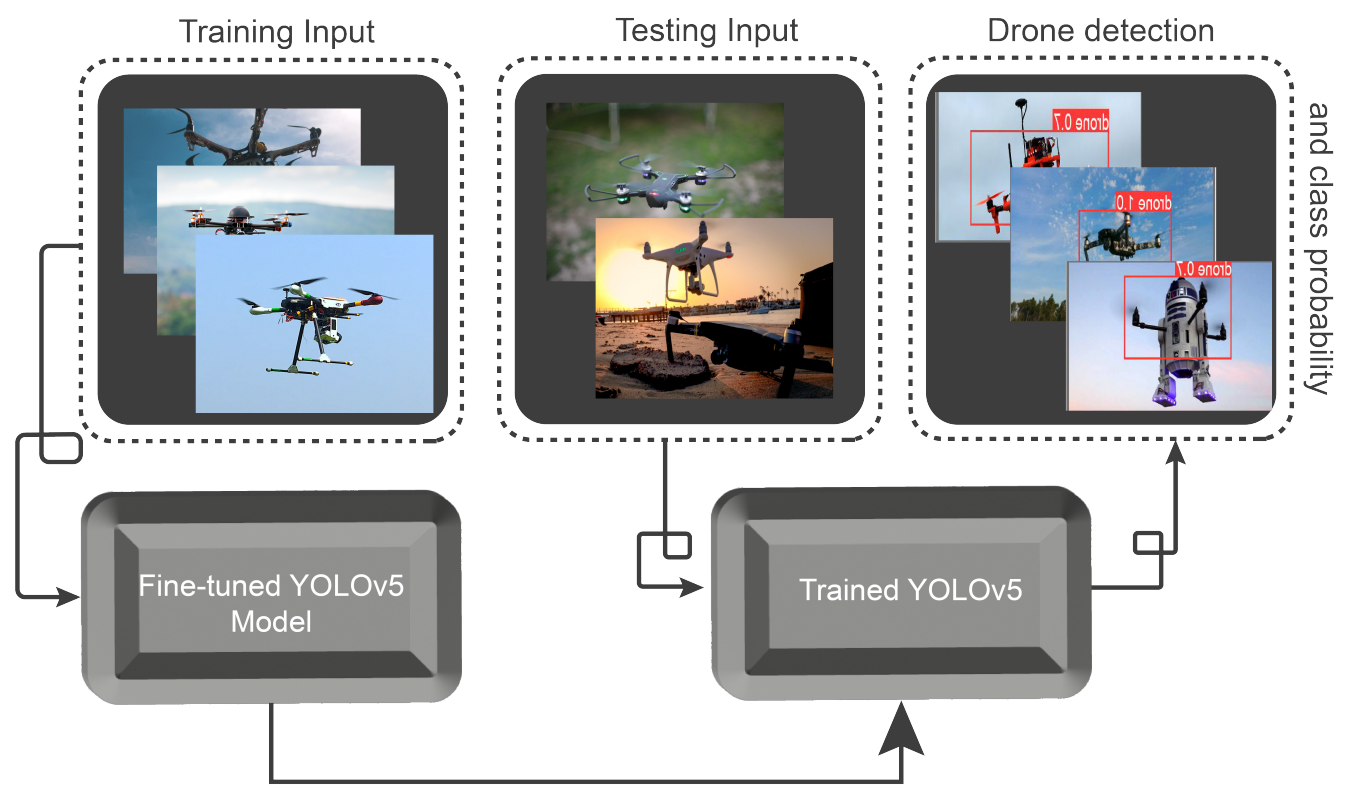

Figure 5. Flowchart of the conducted experiment.
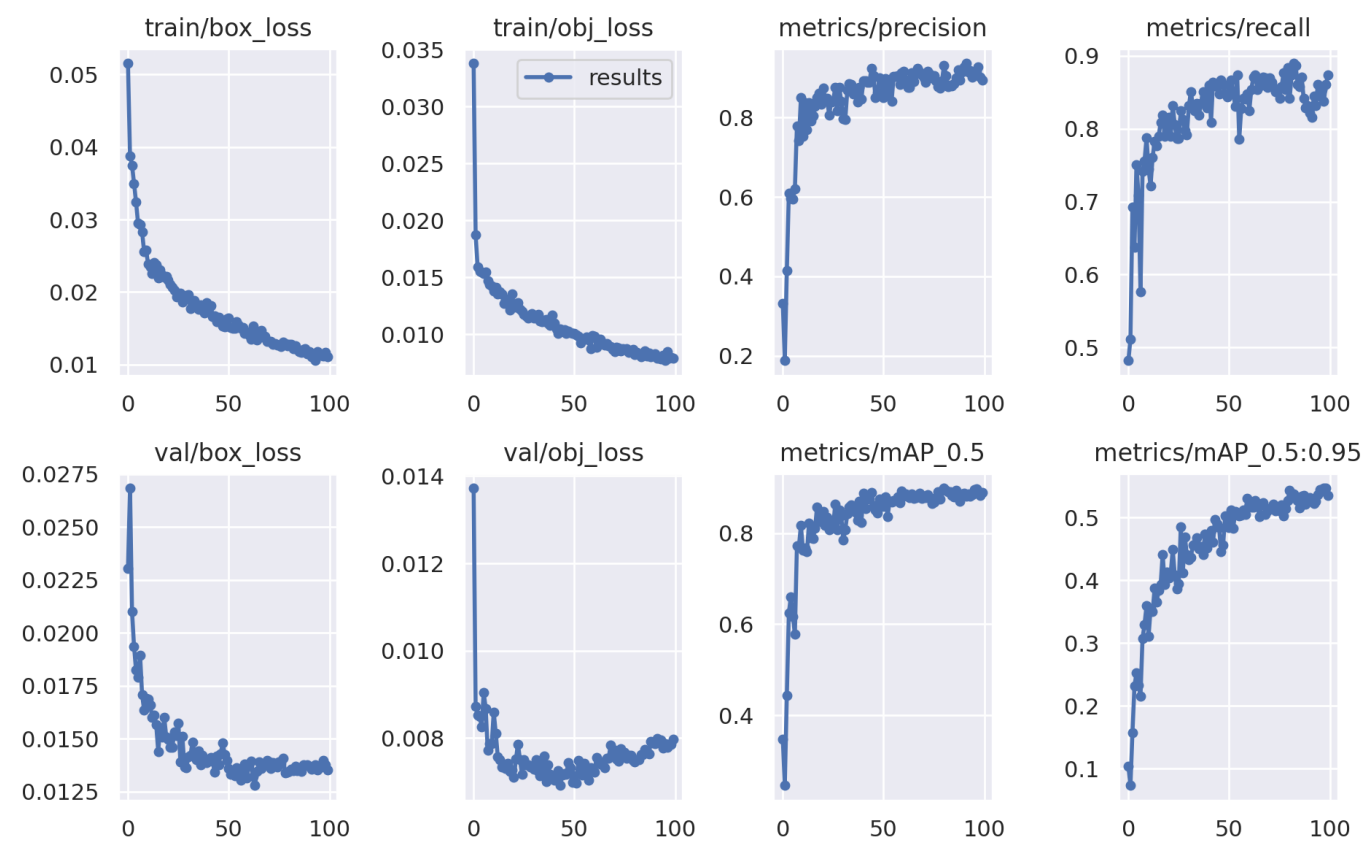

Figure 6. The progress in the performance of the proposed fine-tuned model during the training process.

Figure 6 shows the performance of the fine-tuned YOLOv5 model through the training process. In this figure, the images in the top row refer to the performance of the model for the training set, whereas the images in the bottom row refer to the performance of the model using the validation set. From these images, it can be noted that the loss in detecting drone objects in the training set reached a value less than 0.01 after 100 epochs. However, the loss in detecting drones in the validation set reached its minimum value around epoch number 50; then, it increased slightly as iteration number 100 was approached. As the validation loss kept increasing after the 100th iteration, and to achieve a better generalization of the trained model, we stopped the training process at the 100th iteration.

To further analyze the performance of the model-training process, Figure 7 shows a mapping of the model precision and recall of the drone detection during the training 
process. In the figure, it can be seen that the model could achieve an mAP of $96.8 \%$, which represents the area under the curve. This value refers to the capability of the trained model to accurately detect drone objects with high precision and recall values. To emphasize the generalization of the trained model, the testing set was used to evaluate this model and various criteria were evaluated and compared with those for the other competing models as presented in the next section.

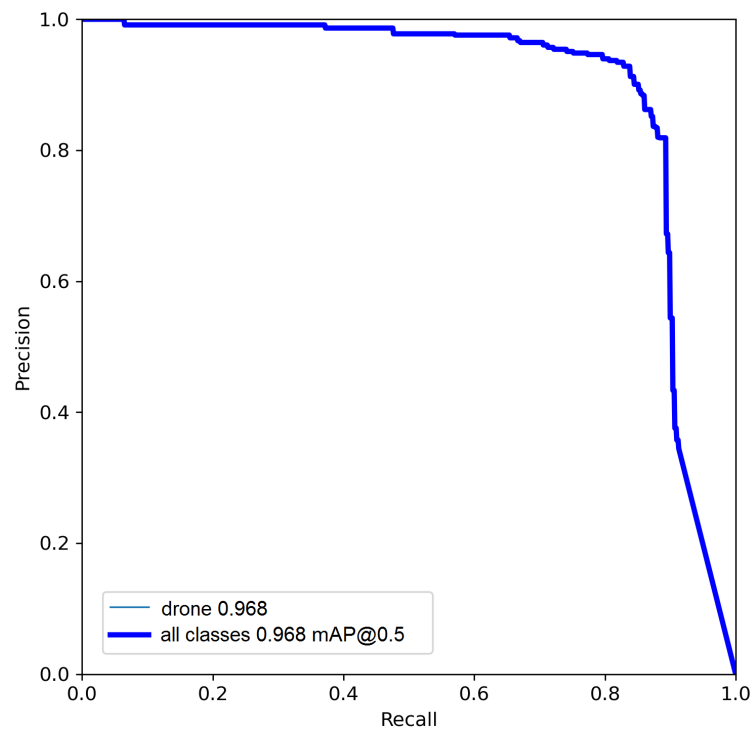

Figure 7. Precision-recall curve for the detection of drone objects in the training set using the proposed fine-tuned model.

Figure 8 presents a set of images containing different types of drone objects along with the detection results using the trained model. As shown in the figure, the proposed approach could accurately detect the locations of drone objects of different types and sizes, which shows the effectiveness of the proposed approach for realizing this task.

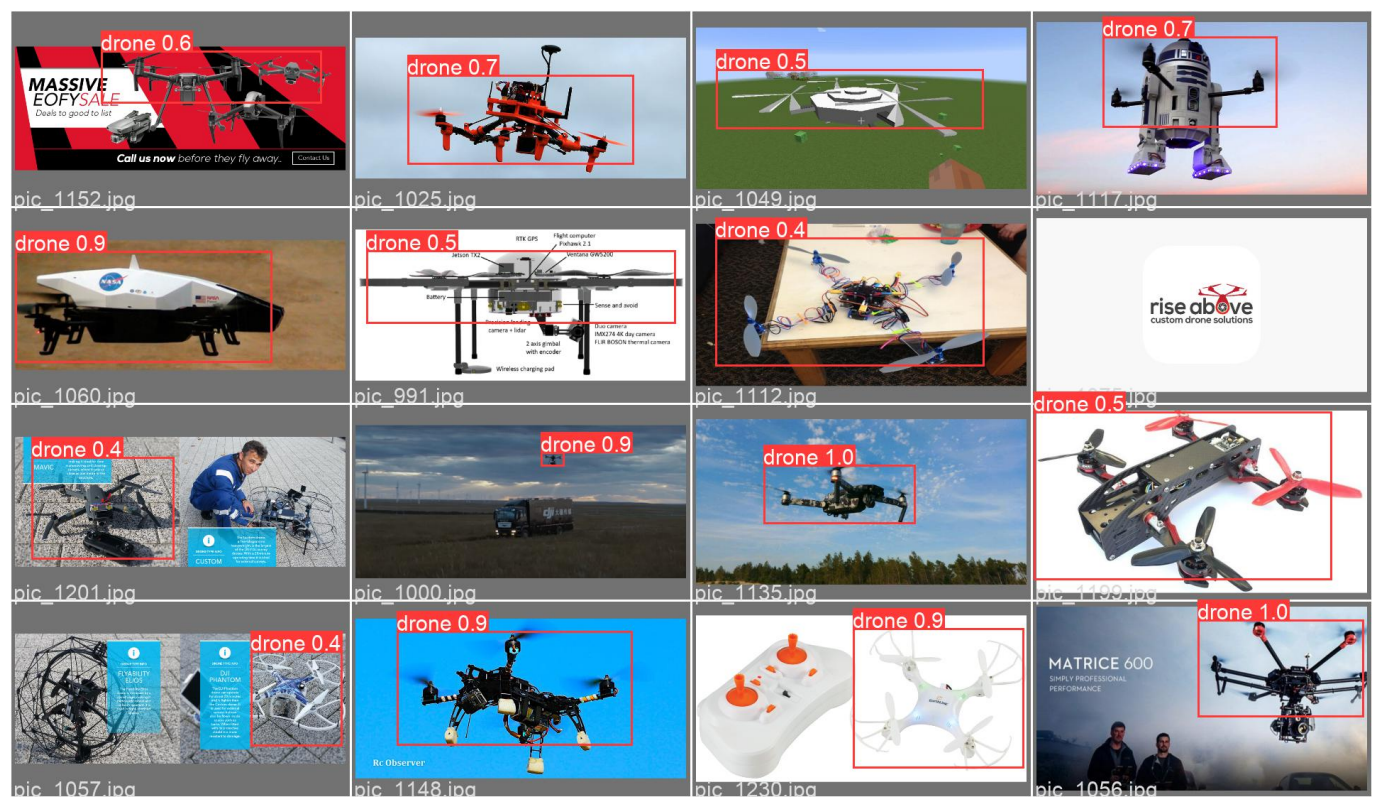

Figure 8. Drone detection using the proposed model supplied with images in the testing set.

\subsection{Comparison with other approaches}

To demonstrate the superiority of the proposed approach for the task of drone detection, several experiments were conducted to measure the performance of other approaches 


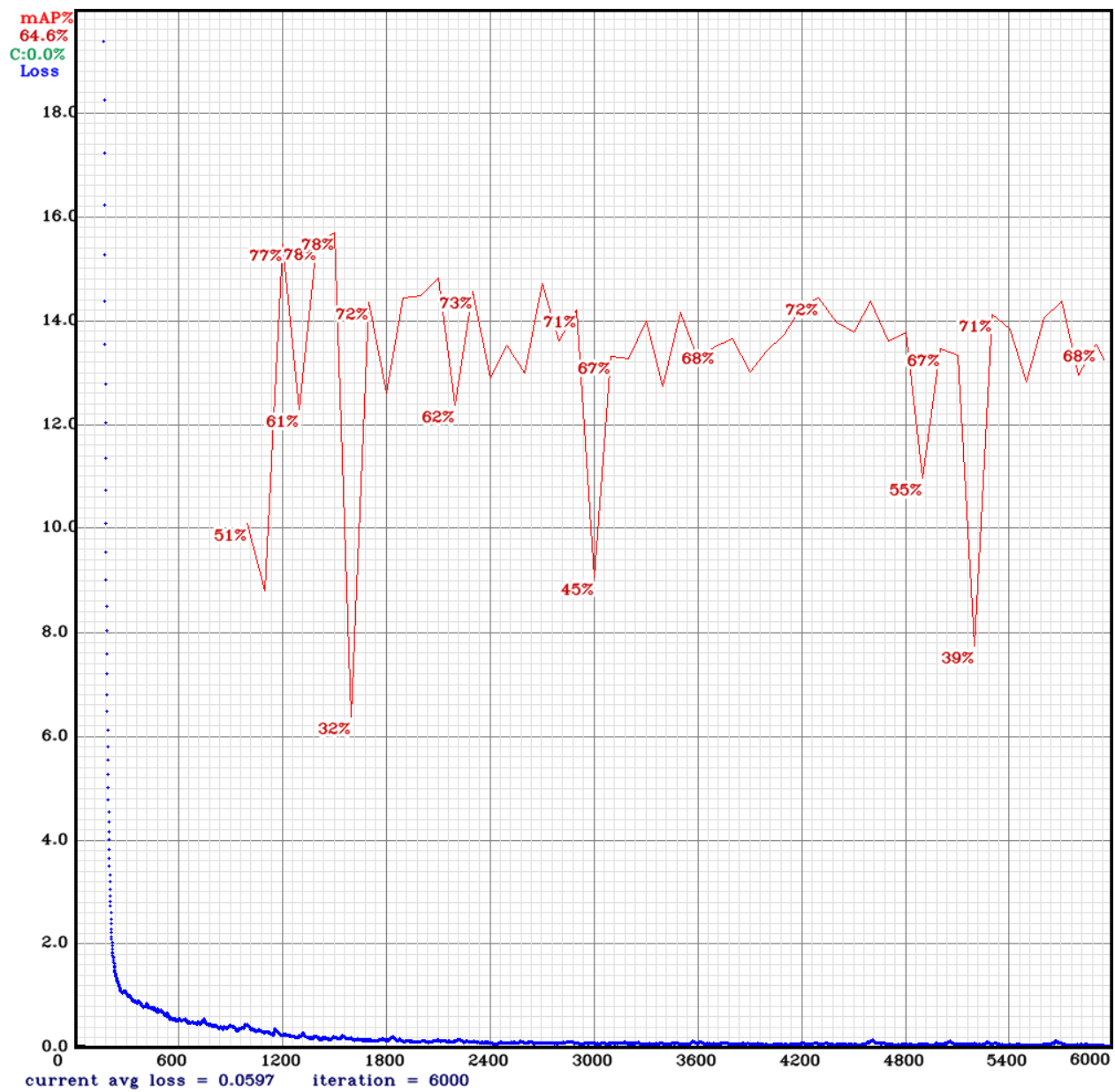

Figure 9. The progress of the loss value while training the approach proposed by Behera and Bazil [41].

such as yolov3 and yolov4 in comparison with the proposed model. Firstly, yolov3 was employed to be trained on the same images of the training and validation sets. Figure 9 shows the progress of the loss and $\mathrm{mAP}$ values while training yolov3 on the training set. As shown in the figure, the training process takes around 6000 iterations to achieve better values of loss and mAP metrics. The minimum achieved average loss value was 0.0597 , and the best achieved mAP was $64.6 \%$ after 6000 iterations. These values represent the mean overall training epochs.

On the other hand, yolov4 was used to be trained on the same dataset, and the progress of the evaluation criteria is depicted in Figure 10. As shown in the figure, the progress achieved by yolov 4 for the $\mathrm{mAP}$ was much better than the corresponding progress achieved by yolov3. The best mAP value achieved by yolov 4 was 89.9 for the training set and, overall, the training epochs. In addition, the best average loss value achieved by yolov 4 was 0.5360 , which is higher than the corresponding value achieved by yolov3. However, the effects of these values can become obvious when we check the generalization of these models.

After training the three models, yolov3, yolov4, and the proposed model, these models were used to check the generalization property. These models were used to measure the evaluation criteria, precision, recall, and $\mathrm{mAP}$ using the training set and testing set. Figure 11 depicts the evaluation results achieved for the three models. As shown in the figure, the results achieved by yolov 4 for the three evaluation criteria are better than those achieved by the other models. However, this did not reflect the generalization of the model. Therefore, the generalization could be checked when we evaluated the trained model on the testing set that the model had not seen before. 


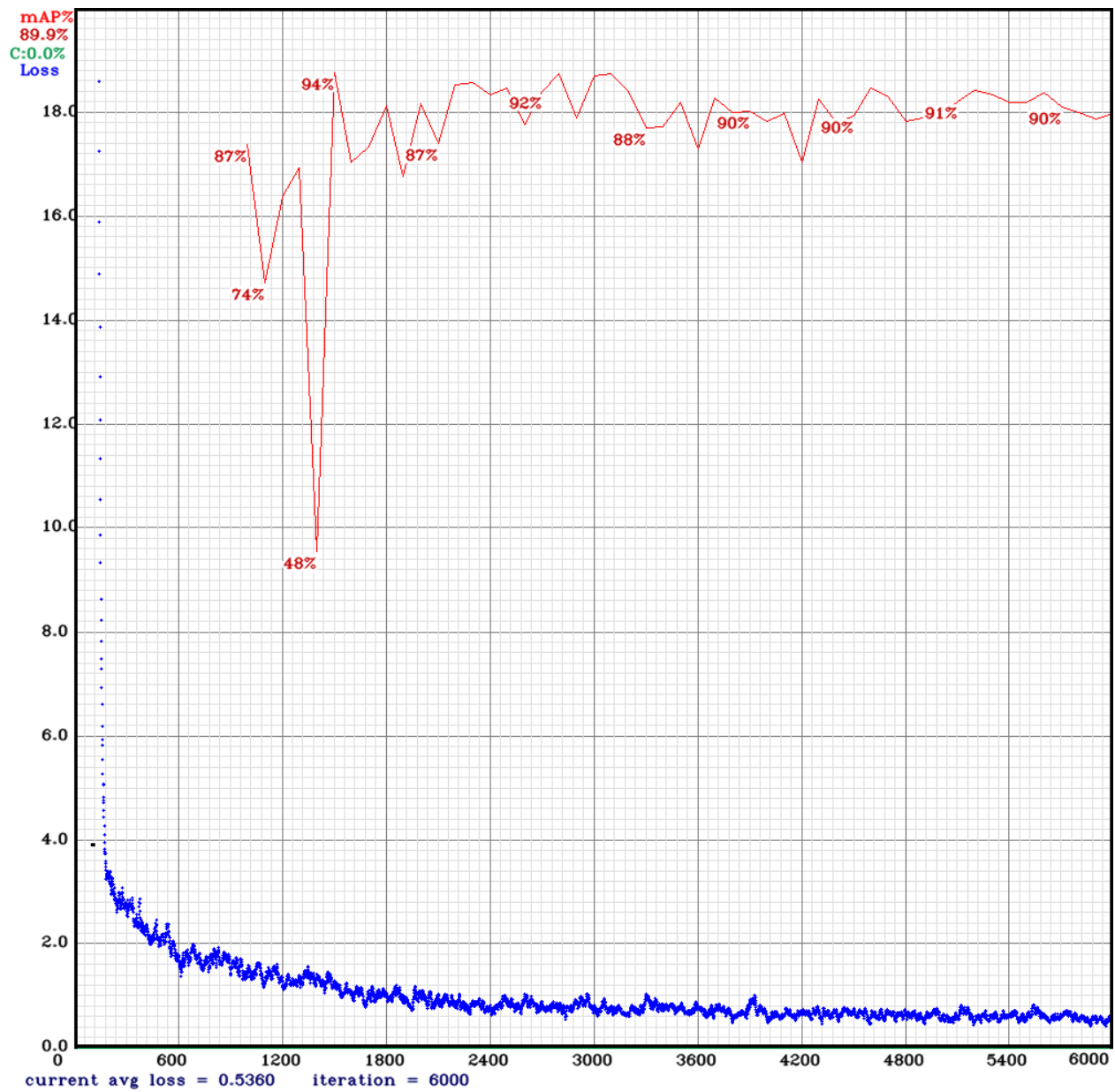

Figure 10. The progress of the loss value while training the method proposed by Tan et al. [42].

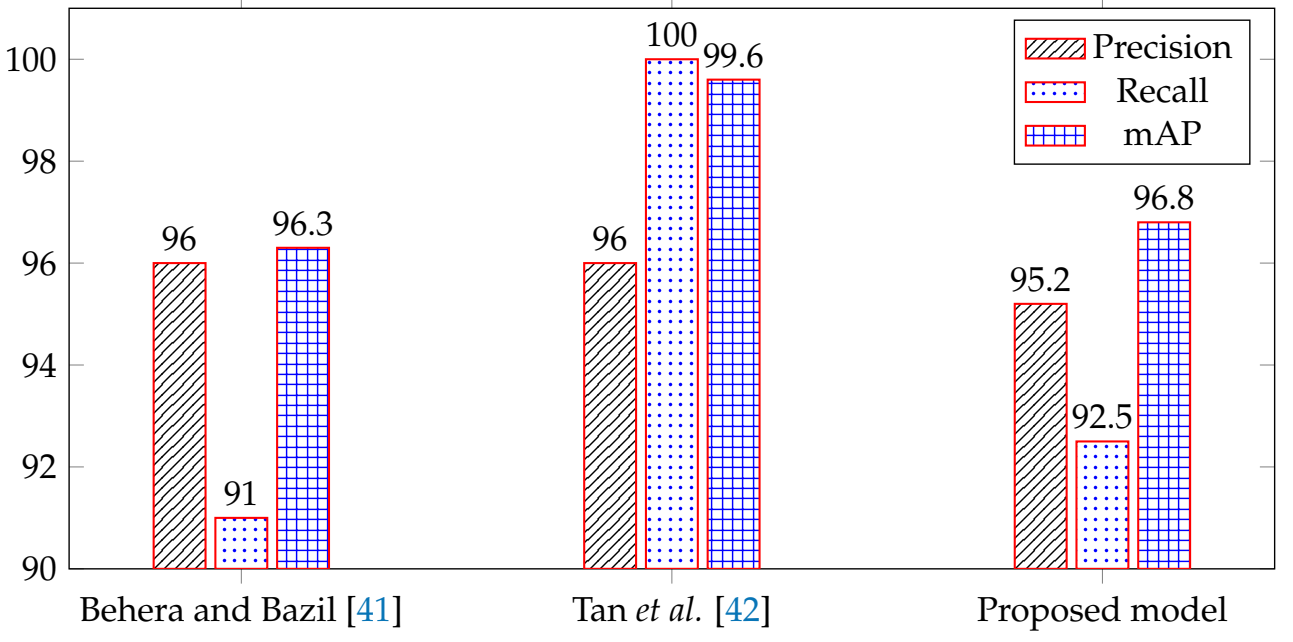

Figure 11. Precision, recall, and mAP results achieved by the trained models for the training set.

As the testing set was used to check the generalization of the trained models, Figure 12 presents the achieved results for the evaluation criteria. In this figure, we can note that, although yolov 4 achieved the best results on the training set, it did not generalize well on the testing set. However, the proposed model could achieve the best generalization and higher values of the evaluation metrics for the testing set. In addition, the precision achieved by yolov3 was better than the precision achieved by yolov 4 for the testing set; however, the recall value of yolov3 was much lower than that of yolov4. On the other hand, 
the proposed model achieved balanced and the best values for the three evaluation criteria. These results emphasize the superiority of the proposed model when compared with the other competing models.

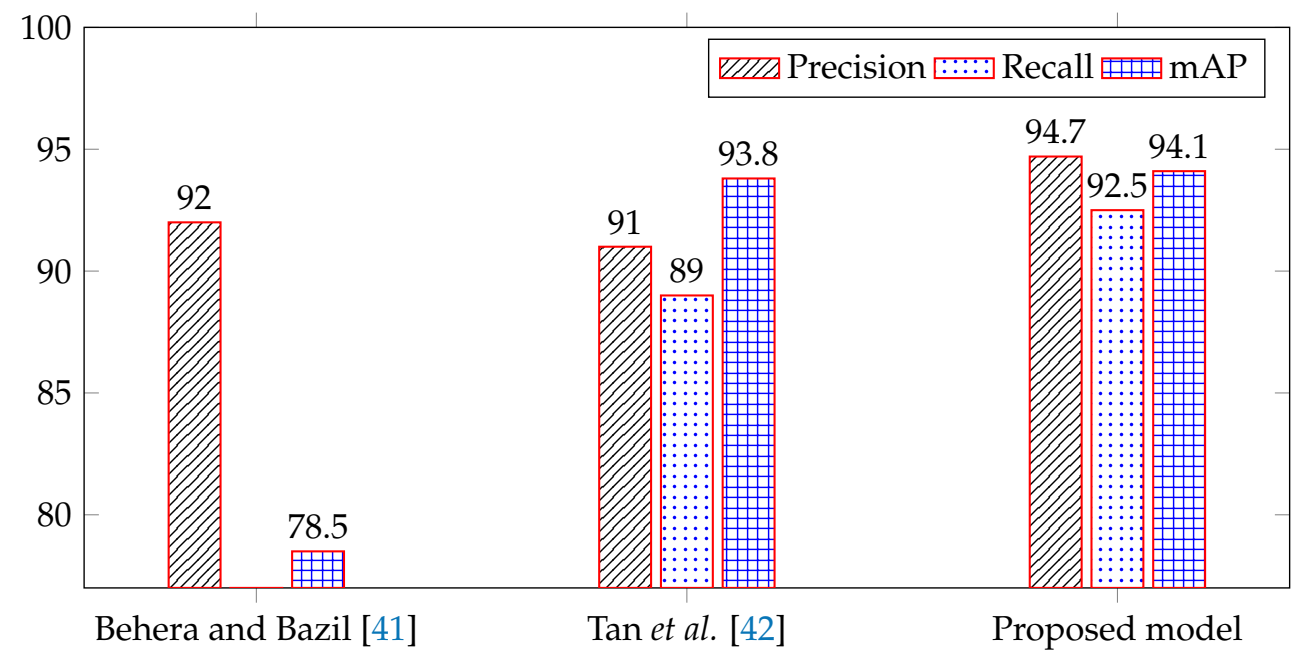

Figure 12. Precision, recall, and mAP results for the testing set using the trained models.
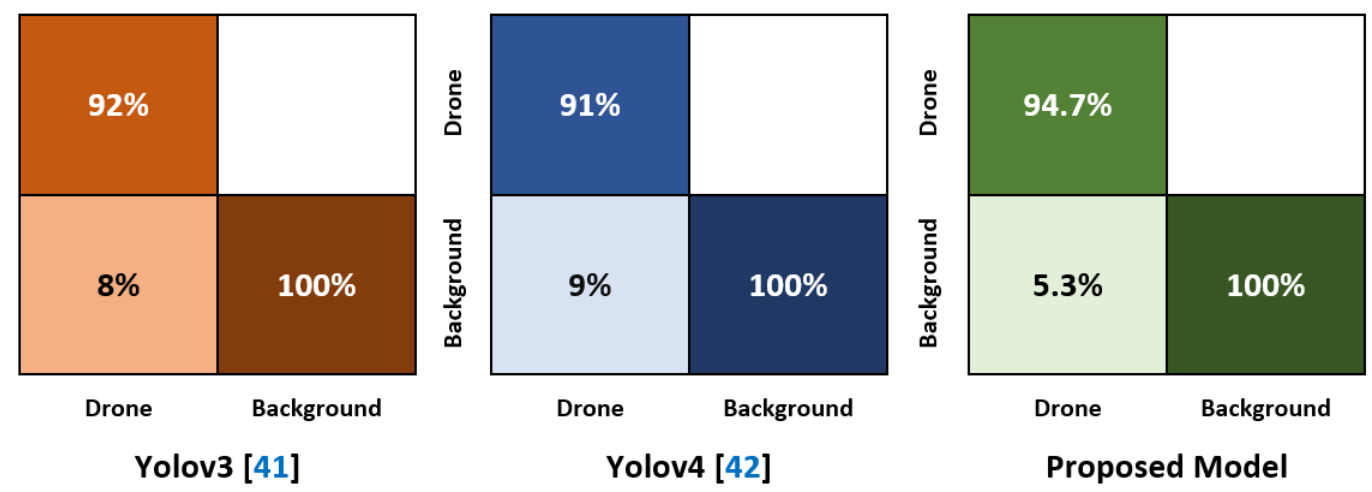

Figure 13. Detection accuracy based on the testing set achieved by [41], [42], and proposed model.

Figure 13 depicts a heatmap of the precision values achieved using the three models on the testing set. In this figure, it can be observed that the precision of the drone detection achieved by yolov 3 and yolov 4 was $92 \%$ and $91 \%$, respectively. However, the precision achieved by the proposed model on the same testing set was $94.7 \%$. These values reflect the efficiency of the proposed model and its promising performance in the task of drone detection.

\section{Future Work}

In the future, we will try to apply our method to a larger dataset to demonstrate the effectiveness of the YOLOv5 algorithm. Moreover, we will include more challenging deadset scenarios and conditions. For example, we will try to have more types of birds, noises, airplanes, and drones. We will also try to have ambiguous images of drones and other objects.

\section{Conclusions}

In this study, we proposed a fine-tuning of the recently emerged yolov5 deep-learning model for detecting drone objects in images captured at various distances. The proposed approach was evaluated using a freely available dataset that consists of different types of drones with different sizes. The fine-tuning was performed by adjusting the model's parameters to best fit the task at hand. In addition, we employed data augmentation to 
provide the training phase of the model with sufficient data for it to be trained properly. The results show that the trained network can detect drones of different types with high accuracy. Moreover, to emphasize the superiority of the proposed approach, two other models, namely, yolov3 and yolov4, were trained and tested on the same dataset, and their results were compared with those for the proposed approach. This comparison showed that the proposed approach outperformed the other models for the adopted evaluation criteria.

Author Contributions: A.R. and B.V., conceptualization, writing, idea proposal, methodology, and results; B.A. and M.A., conceptualization, draft preparation, editing, and visualization; S.A., writing and reviewing; A.A., conceptualization, draft preparation, editing, and reviewing. All the authors have read and agreed to this version of the manuscript.

Funding: This work was partially supported by the Sensor Networks and Cellular System (SNCS) Research Center under Grant 1442-002.

Institutional Review Board Statement: Not applicable.

Informed Consent Statement: Not applicable.

Data Availability Statement: Not applicable.

Acknowledgments: Taif University Researchers Supporting Project number (TURSP-2020/302), Taif University, Taif, Saudi Arabia. The authors gratefully acknowledge the support of the SNCS Research Center at the University of Tabuk, Saudi Arabia. In addition, the authors would like to thank the deanship of scientific research at Shaqra University for supporting this work.

Conflicts of Interest: The authors declare no conflicts of interest.

\section{References}

1. Sandino, J.; Vanegas, F.; Maire, F.; Caccetta, P.; Sanderson, C.; Gonzalez, F. UAV framework for autonomous onboard navigation and people/object detection in cluttered indoor environments. Remote Sensing 2020, 12(20), 3386.

2. Azar, A. T.; Koubaa, A.; Ali Mohamed, N.; Ibrahim, H. A.; Ibrahim, Z. F.; Kazim, M.; ...; Casalino, G. (2021). Drone Deep Reinforcement Learning: A Review. Electronics 2021, 10(9), 999.

3. Udeanu, G.; Dobrescu, A.; Oltean, M. (2016, May). Unmanned aerial vehicle in military operations. In The 18th International Conference "Scientific Research and Education in the Air Force-AFASES", Brasov, Romania, 2014; pp. $199-205$.

4. Pedrozo, S. Swiss military drones and the border space: a critical study of the surveillance exercised by border guards. Geographica Helvetica 2017, 72(1), 97-107.

5. Restas, A. Drone applications for supporting disaster management. World Journal of Engineering and Technology 2015, 3(03), 316.

6. Lee, S.; Choi, Y. Reviews of unmanned aerial vehicle (drone) technology trends and its applications in the mining industry. Geosystem Engineering 2016, 19(4), 197-204.

7. Gallacher, D. Drone applications for environmental management in urban spaces: A review. International Journal of Sustainable Land Use and Urban Planning 2016, 3(4).

8. Saqib, M.; Khan, S. D.; Sharma, N.; Blumenstein, M. A study on detecting drones using deep convolutional neural networks. In 2017 14th IEEE International Conference on Advanced Video and Signal Based Surveillance (AVSS), August, 2017, pp. 1-5. IEEE.

9. Ren, S.; He, K.; Girshick, R.; Sun, J. Faster R-CNN: towards real-time object detection with region proposal networks. IEEE transactions on pattern analysis and machine intelligence 2016, 39(6), 1137-1149.

10. Simonyan, K.; Zisserman, A. Very deep convolutional networks for large-scale image recognition. arXiv preprint arXiv:1409.1556 2014.

11. Aker, C.; Kalkan, S. Using deep networks for drone detection. In 2017 14th IEEE International Conference on Advanced Video and Signal Based Surveillance (AVSS) August, 2017, pp. 1-6. IEEE.

12. Redmon, J.; Farhadi, A. YOLO9000: better, faster, stronger. In Proceedings of the IEEE conference on computer vision and pattern recognition 2017, pp. 7263-7271.

13. Nalamati, M.; Kapoor, A.; Saqib, M.; Sharma, N.; Blumenstein, M. Drone detection in long-range surveillance videos. In $201916 t h$ IEEE International Conference on Advanced Video and Signal Based Surveillance (AVSS), September, 2019, pp. 1-6. IEEE.

14. Liu, W.; Anguelov, D.; Erhan, D.; Szegedy, C.; Reed, S.; Fu, C. Y.; Berg, A. C. Ssd: Single shot multibox detector. In European conference on computer vision, October, 2016, pp. 21-37. Springer, Cham.

15. Szegedy, C.; Vanhoucke, V.; Ioffe, S.; Shlens, J.; Wojna, Z. (2016). Rethinking the inception architecture for computer vision. In Proceedings of the IEEE conference on computer vision and pattern recognition 2016, pp. 2818-2826.

16. He, K.; Zhang, X.; Ren, S.; Sun, J. Deep residual learning for image recognition. In Proceedings of the IEEE conference on computer vision and pattern recognition 2016, pp. 770-778. 
17. Luvizon, D.; Tabia, H.; Picard, D. SSP-Net: Scalable Sequential Pyramid Networks for Real-Time 3D Human Pose Regression. arXiv preprint arXiv:2009.01998 2020.

18. Liu, S.; Qi, L.; Qin, H.; Shi, J.; Jia, J. Path aggregation network for instance segmentation. In Proceedings of the IEEE conference on computer vision and pattern recognition 2018 (pp. 8759-8768).

19. de la Iglesia, D.; Mendez, M.; Dosil, R.; Gonzalez, I. Drone detection CNN for close-and long-range surveillance in mobile applications. Proceedings of the AVSS 2019, Taipei, Taiwan, 18-21.

20. Lin, T. Y.; Goyal, P.; Girshick, R.; He, K.; Dollár, P. Focal loss for dense object detection. In Proceedings of the IEEE international conference on computer vision 2017, pp. 2980-2988.

21. Lin, T. Y.; Dollár, P.; Girshick, R.; He, K.; Hariharan, B.; Belongie, S. Feature pyramid networks for object detection. In Proceedings of the IEEE conference on computer vision and pattern recognition 2017, pp. 2117-2125.

22. He, T.; Zhang, Z.; Zhang, H.; Zhang, Z.; Xie, J.; Li, M. Bag of tricks for image classification with convolutional neural networks. In Proceedings of the IEEE/CVF Conference on Computer Vision and Pattern Recognition 2019, pp. 558-567.

23. Magoulianitis, V.; Ataloglou, D.; Dimou, A.; Zarpalas, D.; Daras, P. Does deep super-resolution enhance uav detection?. In 2019 16th IEEE International Conference on Advanced Video and Signal Based Surveillance (AVSS), September, 2019, pp. 1-6. IEEE.

24. Yamanaka, J.; Kuwashima, S.; Kurita, T. Fast and accurate image super resolution by deep CNN with skip connection and network in network. In International Conference on Neural Information Processing, November, 2017, pp. 217-225. Springer, Cham.

25. Craye, C.; Ardjoune, S. Spatio-temporal semantic segmentation for drone detection. In 2019 16th IEEE International conference on advanced video and signal based surveillance (AVSS), September, 2019, pp. 1-5. IEEE.

26. Schumann, A.; Sommer, L.; Klatte, J.; Schuchert, T.; Beyerer, J. Deep cross-domain flying object classification for robust UAV detection. In 2017 14th IEEE International Conference on Advanced Video and Signal Based Surveillance (AVSS), August, 2017, pp. 1-6. IEEE.

27. Ronneberger, O.; Fischer, P.; Brox, T. U-net: Convolutional networks for biomedical image segmentation. In International Conference on Medical image computing and computer-assisted intervention, October, 2015, pp. 234-241. Springer, Cham.

28. Seidaliyeva, U.; Akhmetov, D.; Ilipbayeva, L.; Matson, E. T. Real-Time and accurate drone detection in a video with a static background. Sensors 2020, 20(14), 3856.

29. Khan, F. R.; Muhabullah, M.; Islam, R.; Monirujjaman Khan, M.; Masud, M.; Aljahdali, S.; ... Singh, P. A Cost-Efficient Autonomous Air Defense System for National Security. Security and Communication Networks 2021, 2021.

30. Yao, J.; Qi, J.; Zhang, J.; Shao, H.; Yang, J.; Li, X. A Real-time detection algorithm for kiwifruit defects based on YOLOv5. Electronics 2021, 10(14), 1711.

31. Wu, W.; Liu, H.; Li, L.; Long, Y.; Wang, X.; Wang, Z.; ... Chang, Y. Application of local fully Convolutional Neural Network combined with YOLO v5 algorithm in small target detection of remote sensing image. PloS one 2021, 16(10), e0259283.

32. Redmon, Joseph, Santosh Divvala, Ross Girshick, and Ali Farhadi. "You only look once: Unified, real-time object detection." In Proceedings of the IEEE conference on computer vision and pattern recognition, pp. 779-788. 2016

33. Redmon, Joseph, and Ali Farhadi. "YOLO9000: better, faster, stronger." In Proceedings of the IEEE conference on computer vision and pattern recognition, pp. 7263-7271. 2017.

34. Redmon, Joseph, and Ali Farhadi. "Yolov3: An incremental improvement." arXiv preprint arXiv:1804.02767 (2018).

35. Bochkovskiy, Alexey, Chien-Yao Wang, and Hong-Yuan Mark Liao. "Yolov4: Optimal speed and accuracy of object detection." arXiv preprint arXiv:2004.10934 (2020).

36. Qi, Delong, Weijun Tan, Qi Yao, and Jingfeng Liu. "YOLO5Face: Why Reinventing a Face Detector." arXiv preprint arXiv:2105.12931 (2021).

37. Zhang, Shifeng, Cheng Chi, Zhen Lei, and Stan Z. Li. "Refineface: Refinement neural network for high performance face detection." IEEE Transactions on Pattern Analysis and Machine Intelligence (2020).

38. Github. Yolov5. Available online: https://github.com/ultralytics/yolov5, (accessed on 9/12/2021).

39. Mehdi Ozel. Available online: https://www.kaggle.com/dasmehdixtr/drone-dataset-uav, (accessed on 25/12/2021).

40. Xu, Qingqing, et al. "Effective Face Detector Based on YOLOv5 and Superresolution Reconstruction." Computational and Mathematical Methods in Medicine 2021 (2021).

41. D. Behera and A. Bazil, "Drone Detection and Classification using Deep Learning," in International Conference on Intelligent Computing and Control Systems (ICICCS), pp. 1012-1016 (2020).

42. L. Tan, L. Xinyue, L. Xiaofeng, G.Wang, "YOLOv4 Drone: UAV image target detection based on an improved YOLOv4 algorithm," Computers \& Electrical Engineering, vol. 93, pp. 107261 (2021).

43. Xu Q, Zhu Z, Ge H, Zhang Z, Zang X. Effective Face Detector Based on YOLOv5 and Superresolution Reconstruction. Computational and Mathematical Methods in Medicine. Nov 16;2021. 